\title{
Sleep Deprivation Amplifies Reactivity of Brain Reward Networks, Biasing the Appraisal of Positive Emotional Experiences
}

\author{
Ninad Gujar, ${ }^{1}$ Seung-Schik Yoo, ${ }^{2}$ Peter Hu, ${ }^{1}$ and Matthew P. Walker ${ }^{1}$ \\ ${ }^{1}$ Sleep and Neuroimaging Laboratory, Department of Psychology and Helen Wills Neuroscience Institute, University of California, Berkeley, Berkeley, \\ California 94720-1650, and 2Department of Radiology, Brigham and Women's Hospital, Harvard Medical School, Boston, Massachusetts 02115
}

\begin{abstract}
Appropriate interpretation of pleasurable, rewarding experiences favors decisions that enhance survival. Conversely, dysfunctional affective brain processing can lead to life-threatening risk behaviors (e.g., addiction) and emotion imbalance (e.g., mood disorders). The state of sleep deprivation continues to be associated with maladaptive emotional regulation, leading to exaggerated neural and behavioral reactivity to negative, aversive experiences. However, such detrimental consequences are paradoxically aligned with the perplexing antidepressant benefit of sleep deprivation, elevating mood in a proportion of patients with major depression. Nevertheless, it remains unknown how sleep loss alters the dynamics of brain and behavioral reactivity to rewarding, positive emotional experiences. Using functional magnetic resonance imaging (fMRI), here we demonstrate that sleep deprivation amplifies reactivity throughout human mesolimbic reward brain networks in response to pleasure-evoking stimuli. In addition, this amplified reactivity was associated with enhanced connectivity in early primary visual processing pathways and extended limbic regions, yet with a reduction in coupling with medial frontal and orbitofrontal regions. These neural changes were accompanied by a biased increase in the number of emotional stimuli judged as pleasant in the sleep-deprived group, the extent of which exclusively correlated with activity in mesolimbic regions. Together, these data support a view that sleep deprivation not only is associated with enhanced reactivity toward negative stimuli, but imposes a bidirectional nature of affective imbalance, associated with amplified reward-relevant reactivity toward pleasure-evoking stimuli also. Such findings may offer a neural foundation on which to consider interactions between sleep loss and emotional reactivity in a variety of clinical mood disorders.
\end{abstract}

\section{Introduction}

Optimal evaluation and interpretation of pleasurable, rewarding experiences allows for the development of actions and decisions toward fitness-enhancing outcomes (Berridge and Robinson, 2003; Schultz, 2006; Knutson and Wimmer, 2007). However, pleasure seeking can also lead to deleterious and life-threatening behaviors, exemplified by abusive drug addiction, impulsive thrill seeking, and adverse risk taking. Experimental paradigms have demonstrated that these reward mechanisms are supported, in part, by activity in mesolimbic reward pathways including the ventral tegmental nuclei and striatum (Schultz, 2006), as well as the amygdala (Berridge and Robinson, 2003; Murray, 2007). One circumstance increasingly related to maladaptive emotional and mood regulation is the state of sleep deprivation. Building on extensive clinical findings (Benca et al., 1992; Armitage, 2007; Harvey, 2008), a growing literature continues to emphasize the

Received June 22, 2010; revised Dec. 23, 2010; accepted Jan. 21, 2011.

This work was supported in part by grants from the National Institutes of Health [National Institute on Aging Grant AG31164 (M.P.W.)] and the University of California, Berkeley (M.P.W.). We thank Edwin Robertson and Jonathan Wallis for their thoughtful insights and comments on this work.

Correspondence should be addressed to Matthew P. Walker, Department of Psychology, Tolman Hall 3331, University of California, Berkeley, Berkeley, CA 94720-1650. E-mail: mpwalker@berkeley.edu.

DOI:10.1523/JNEUROSCI.3220-10.2011

Copyright $\odot 2011$ the authors $\quad 0270-6474 / 11 / 314466-09 \$ 15.00 / 0$ necessity of sleep in the optimal homeostasis of emotional brain function (Dahl, 1996; Levin and Nielsen, 2009; Walker and van der Helm, 2009). Chronic sleep restriction has been shown to increase mood disturbance and escalate complaints of emotional difficulties (Dinges et al., 1997; Zohar et al., 2005). Moreover, acute sleep deprivation significantly amplifies amygdala reactivity in response to negative emotional stimuli, associated with a loss of medial prefrontal cortex (mPFC) connectivity (Yoo et al., $2007 b$ ). Sleep loss also disrupts consolidation of negative emotional memories, resulting in persistent amygdala reactivity during later recollection (Sterpenich et al., 2007). Furthermore, larger pupillary responses have been reported under conditions of sleep deprivation, specifically during the viewing of negative emotional picture stimuli (Franzen et al., 2009).

This detrimental affective consequence of a lack of sleep is, however, paradoxically aligned with the beneficial antidepressant effect of sleep deprivation in patients with major depression, a substantial proportion of whom show a fast-acting alleviation of depressed mood in response to total or selective sleep deprivation (Gillin et al., 2001). Unfortunately, the antidepressant benefit of sleep deprivation is rapidly reversed following recovery sleep, implying a transient (functional) basis for the short-term clinical improvement (Wirz-Justice and Van den Hoofdakker, 1999). Interestingly, this beneficial mood-elevating effect is paralleled by reports of emotional lability in healthy adults under conditions of 
sleep loss, commonly describing episodes of inappropriate euphoria and giddiness, and oscillating periods of lopsided positive emotional reactivity (Bliss et al., 1959; Horne, 1993; Dahl, 1996).

These findings contribute to the hypothesis that sleep deprivation may be associated not only with enhanced reactivity toward threat-relevant negative stimuli (Yoo et al., 2007b), but also a disinhibition of mesolimbic dopaminergic networks that mediate reactivity to pleasurable and rewarding experiences (Venkatraman et al., 2007; Volkow et al., 2008, 2009). However, to date, the assessment of emotional brain reactivity under conditions of sleep deprivation has focused on negatively valenced stimuli. It remains unclear whether this relationship is unidirectional, associated with selective overresponding by the brain only to negative stimuli, or whether the impact of sleep loss imposes a labile, bidirectional nature of affective imbalance, including increased neural reactivity to positive emotional experiences also. Here, we test the hypothesis that sleep deprivation amplifies reactivityand alters functional connectivity-within mesolimbic networks of the human brain in response to pleasure-evoking stimuli, biasing the appraisal — and hence the behavioral ratings— of these positive emotional experiences.

\section{Materials and Methods}

Twenty-seven healthy participants age 18-30 years (mean 23.6, s.d. \pm 1.4 ) were assigned to either a sleep-deprivation group ( $n=14,7$ males) or sleep-control group ( $n=13,6$ males). The experimental intervention differentiating the two groups occurred on the night before functional magnetic resonance imaging (fMRI)-task scanning. In the sleepdeprivation group, subjects were awake across day 1 , night 1 , and day 2 , before fMRI scanning on day 2 (5:00 P.M.). In the sleep-control group, subjects slept normally at home across night 1 before scanning on day 2 (5:00 P.M.).

Participants abstained from caffeine, alcohol, and naps for $72 \mathrm{~h}$ before and during the study, and kept a normal sleep-wake rhythm and average sleep duration (7-9 h of sleep per night, morning rise time of 7:00-8:30 A.M.) for 1 week before study participation, verified by actigraphy (a wristwatch movement sensor, sensitive to wake and sleep states). The amount of sleep obtained during the period before the study did not differ between the sleep-deprivation group and the sleep-rested group in either average number of hours (mean: 7.75 and 7.61, respectively: twosample $t$ test, $t_{(25)}=0.28, p=0.78$ ) or variability, as assessed in standard deviation across nights (mean: 0.46 and 0.53 , respectively: $t_{(25)}=0.66$, $p=0.43$ ). Exclusion criteria, assessed using a prescreening questionnaire, were a history of sleep disorders, neurologic disorders or closed head injury, Axis I psychiatric disorders according to the DSM-IV criteria (encompassing major mental disorders including depression, anxiety disorders, bipolar disorder, attention deficit disorder, and schizophrenia), history of drug abuse, and current use of anti-depressant or hypnotic medication. No subject reported being a habitual napper (defined as napping 2 or more days per week).

Subjects in the deprivation group were continuously monitored in the laboratory throughout the enforced waking period by trained personnel, independently confirmed using actigraphy monitoring, accumulating a mean of 31.9 h (s.d. \pm 1.31 ) total sleep deprivation before the scanning session. A standardized regimen of waking activity was conducted during the deprivation period, with subject activities limited to Internet, e-mail, and reading in a standard light environment (340 lux). Participants were allowed occasional short indoor walks around the floor of the experimental area (maximum light: 530 lux). During the deprivation period, a standard breakfast was provided to each subject (7:00 A.M.), which contained no caffeine and included yogurt or cereal, toast, a small fruit bowl, and a glass of orange juice ( $\sim 400$ calories), and a standard lunch was provided (12:00 P.M.) containing a sandwich (variable filling, depending on subjects' dietary preferences), a small bag of pretzels, and a granola bar ( $\sim 550$ calories). Water intake was offered ad libitum throughout the experimental period, as were snack options of either a small box of dried raisins or bag of pretzels.
Participants in the sleep-control group obtained a mean of $7.7 \mathrm{~h}$ (s.d. \pm 1.23 ) of sleep the night before the fMRI session, as measured using actigraphy. On the night before deprivation, participants in the sleepdeprivation group obtained a mean of $7.4 \mathrm{~h}$ (s.d. \pm 0.98 ) of sleep, not significantly different from that of the control group $(7.1 \mathrm{~h}$; s.d. \pm 1.11 : $\left.t_{(25)}=0.75, p=0.46\right)$. It should be noted that these are quantitative measures and, due to the absence of polysomnographic sleep assessments, do not inform about qualitative sleep-stage measures. The study protocol was approved by the institutional ethical review board, and all participants provided written informed consent.

$f M R I$ and task procedures. Functional images were collected on a General Electric 3 tesla magnet using an echo planar imaging sequence $(64 \times$ 64 matrix; $\mathrm{TR}=2500 \mathrm{~ms} ; \mathrm{TE}=40 \mathrm{~ms} ; \mathrm{FOV}=240 \mathrm{~cm}$; oblique slice parallel to AC-PC line, 34 slices, no slice gap, $4 \mathrm{~mm}$ thickness), followed by a high-resolution, T1-weighted structural scan (3-dimensional spoiled gradient echo sequence, $256 \times 192$ matrix; TR $=20 \mathrm{~ms}$; TE $=$ minimum; flip angle $=30^{\circ}$; FOV $=240 \mathrm{~cm} ; 124$ slices; $1.3 \mathrm{~mm}$ thickness). All images were corrected for slice timing, realigned, coregistered, normalized, and smoothed [ $8 \mathrm{~mm}$ full-width at half-maximum (FWHM)] using default parameters in SPM5.

The event-related fMRI design was composed of 100 picture stimulus presentation trials, selected from a standardized picture set (International Affective Picture System) (Lang, 1997). The picture stimuli ranged in a standardized gradient from emotionally neutral (neutral valence, low arousal) to increasingly pleasant (positive valence, high arousal; 100 stimulus-set range: valence: maximum $=8.28$, minimum $=3.79$, mean $=6.18$, s.d. \pm 1.30 ; arousal: maximum $=6.99$, minimum $=3.14$, mean $=4.88$, s.d. \pm 1.07$)$. Each event-related trial lasted $10 \mathrm{~s}$, beginning with a fixation cross (300-900 ms jittered), followed by a picture stimulus presentation $(2500 \mathrm{~ms})$. Following this stimulus, participants subsequently made a binary button-press response judgment, categorizing the picture stimulus as either emotionally "pleasant" or "neutral" (2500 ms), which further confirmed wakefulness. The trial was completed by $4100-$ $4700 \mathrm{~ms}$ fixation (equating jitter time).

Behavioral data analysis. Participants' task responses provided two related behavioral outcome measures. The first was the proportion of picture stimuli categorized by participants as either "pleasant" or "neutral." The second was a positive bias score, which used these values, and represented the subtracted difference in the proportion of picture stimuli categorized by the participants as "neutral" from the proportion of picture stimuli categorized as "pleasant." A zero value therefore represents equal numbers of stimuli assigned to neutral and pleasant categories, while a positive value represents a greater proportion of stimuli rated as pleasant relative to neutral (and vice versa).

Response data (categorization as pleasant or neutral) were submitted to a mixed-design ANOVA, with between-subjects factor "group" (sleep control, sleep deprivation), and within-subjects factor "emotional response" (pleasant, neutral). Post hoc within- and between-group comparisons of response categorizations and the positive response bias were calculated using paired and unpaired two-tailed $t$ tests, respectively. All analyses were performed using the software program JMP v8.0 (SAS), with $p<0.05$ considered significant.

$f M R I$ analysis. Preprocessing and data analysis were performed using Statistical Parametric Mapping software implemented in Matlab (SPM5; Wellcome Department of Cognitive Neurology, London, UK). Images were slice-timing corrected and motion corrected, and then spatially normalized to the Montreal Neurological Institute template and smoothed using an $8 \mathrm{~mm}$ FWHM Gaussian kernel. For each subject, trial-related activity was assessed by convolving a vector of trial onsets with a canonical hemodynamic response function. The six movementrelated variances (three rigid-body translations and three rotations, determined from the realignment preprocessing step) were used as regressors in the design matrix for modeling movement-related artifact in the time series. Nonsphericity of the error covariance was accommodated for by a first-order autoregressive (AR1) model, in which the temporal autocorrelation was estimated by pooling over suprathreshold voxels (Friston et al., 2002).

A general linear model (GLM) (Friston et al., 1995) was specified for each participant to investigate the effects of interest. Contrasts were cre- 
ated at the first level using parametric modulation (Büchel et al., 1998), integrating the standardized valence score of each individual picture slide, allowing the elucidation of brain activation that covaried with this regressor (positive emotional strength), and resulting in a corresponding $t$ statistic for every voxel. This analysis method afforded an objective evaluation of brain responsivity to the standardized picture slides, quantifying activation in accordance with the associated normative stimulus ratings, which were uniformly applied to both groups. The resulting contrasts were then taken through to a second-level random-effects analysis to assess group-level differences. Omitted response trials were modeled separately, and did not differ significantly in number between the sleep-control and sleep-deprivation groups [mean: 0.62 (s.d. \pm 0.75$)$ and 1.07 (s.d. \pm 2.26 ), respectively, two-sample $t$ test, $t_{(25)}=0.89, p=0.38$ ].

Functional connectivity was assessed using psychophysiological interaction analysis, implemented in SPM5, evaluating how regional network activity covaries in relation to a source region during task performance (Friston et al., 1997). Within each group, we examined functional connectivity referenced to a $4 \mathrm{~mm}^{3}$ sphere seed region of interest (ROI) at peak locations of group difference. A GLM was constructed at the first level using three regressors: (1) the deconvolved bold signal from the ROI seed, (2) the stimulus valence scores, and (3) the interaction term between the first and the second regressor. Contrasts for this interaction term revealed brain regions considered to covary as a functional network with the source ROI (Friston et al., 1997). These subsequent connectivity contrasts were then taken through to a second-level, random-effects analysis.

The relationship between the positive emotional bias score and brain activity within each group was identified by GLM using a simple regression analysis, and also reported at a significance threshold at $p<0.001$, whole-brain uncorrected, and $\geq 5$ contiguous voxels. Formal estimation of overlap in activation patterns used conjunction analysis applied to the statistical parametric maps of the minimum $t$ statistic over two selected contrasts, using the conjunction null hypothesis test (Nichols et al., 2005), which requires regions of significance to be present in both tested conditions (i.e., a logical "AND” conjunction).

For our a priori ROIs (midbrain, striatum, amygdala, and insula) (Berridge and Robinson, 2003; Adcock et al., 2006; Schultz, 2006; Hampton et al., 2007; Knutson and Wimmer, 2007; Murray, 2007; Yoo et al., 2007; Shohamy and Wagner, 2008; Düzel et al., 2009; Valentin and O'Doherty, 2009), statistical inferences are reported using small volume correction (SVC) at a threshold of $p<0.05$, corrected (see Table 1). All ROIs used a $10 \mathrm{~mm}^{3}$ sphere, with the exception of the smaller ventral tegmental area (VTA)/substantia nigra structure, set instead at $5 \mathrm{~mm}^{3}$ (Adcock et al., 2006; D’Ardenne et al., 2008; Düzel et al., 2009). Whole-brain analyses beyond these ROIs are reported using significance level of $p<0.001$ whole-brain uncorrected, with a spatial threshold of $\geq 5$ voxel cluster size, as in previous such studies [e.g., Pochon et al. (2002), Delgado et al. (2005), Wittmann et al. (2005, 2008a,b), Adcock et al. (2006), Smith et al. (2006), Chib et al. (2009), Guitart-Masip et al. (2010), and Kuhl et al. (2010)], although importantly we note in the results which of these non-a priori regions survived the more conservative false discovery rate correction $(p<0.05)$. This approach provides a reasonable balance with respect to type I and type II error concerns, consistent with the false discovery rate in typical behavioral science research, as outlined by Lieberman and Cunningham (2009).

\section{Results}

\section{Behavior}

The distribution of responses categorizing stimuli as either pleasant or neutral, displayed in Figure $1 A$, demonstrated no main effect of group (sleep control, sleep deprivation) or emotional response (neutral, positive) (significance provided in figure), yet a significant group $\times$ emotional response interaction $\left(F_{(1,25)}=\right.$ 4.09, $p=0.04)$. Post hoc tests demonstrated a disproportionate positive response bias in the sleep-deprivation group. Specifically, relative to the null hypothesis of equal categorical assignment (50/50), sleep-deprived participants rated significantly more images as pleasant, and significantly fewer images as neutral

Table 1. Anatomical coordinates for regions of significant difference between the sleep control and sleep deprivation groups in response to the emotional stimulus gradient

\begin{tabular}{llllllll}
\hline Region & $\begin{array}{l}\text { Cluster size } \\
\text { (voxels) }\end{array}$ & $x$ & $y$ & $z$ & Zscore & $p$ \\
\hline
\end{tabular}

Sleep deprivation $>$ sleep control

Amygdala ${ }^{a, b, c}$

L $\quad 47$

$\mathrm{R}$

Insular cortex- $\mathrm{L}^{a, b, c, d}$

Putamen- $\mathrm{L}^{b, d, e}$

Substantia nigra/ventral tegmental area, ${ }^{b, d, f, g, h}$

Fusiform gyrus - $L^{*}$

Sleep control $>$ sleep deprivation

Posterior hippocampal cortex- $R^{*}$

Precuneus- $L^{*}$

Middle occipital gyrus - $L^{*}$
47

12

20

9

9

46

7

$\begin{array}{llll}30 & -28 & -9 & 3.51\end{array}$

8

5

$\begin{array}{rrrr}-24 & -9 & -21 & 3.65 \\ 30 & 3 & -18 & 3.39 \\ -44 & 4 & 3 & 3.60 \\ -30 & 5 & -2 & 3.62 \\ 0 & -21 & -18 & 3.51\end{array}$

0.012

0.032

0.027

0.026

0.007

The $x-y-z$ coordinates are given in peak Montreal Neurological Institute (MNI) space coordinates. L and R denote left and right. * denotes non-a priori regions that were significant at $p<0.001$ uncorrected threshold, but did not survive whole-brain FDR correction at $p<0.05$. All other areas were of a priori interest, defined by letter $(a-g)$, and all survived SVC correction for multiple comparisons ( $p<0.05,10 \mathrm{~mm}^{3}$ sphere; with the exception of the small substantia nigra/VTA nuclei, which used a $5 \mathrm{~mm}^{3}$ sphere).

${ }^{a}$ Karama et al. (2002)

${ }^{b}$ Gottfried et al. (2003).

'Sabatinelli et al. (2007).

${ }^{d}$ Knutson et al. (2005).

eValentin and $0^{\prime}$ Doherty (2009).

${ }^{f}$ Adcock et al. (2006).

${ }^{g}$ Kuhl et al. (2010).

${ }^{h} D^{\prime}$ Ardenne et al. (2008).
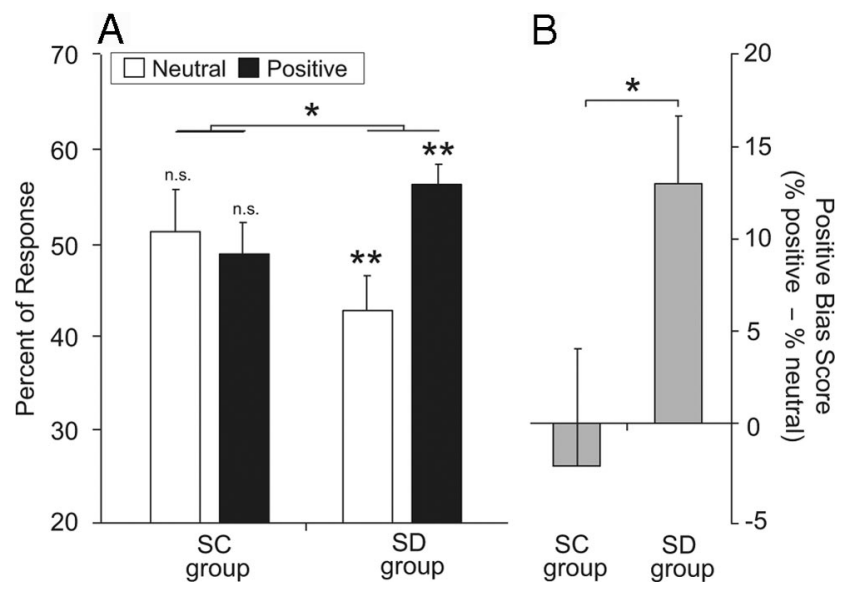

Figure 1. Stimulus ratings. $A$, The proportion of stimuli categorized as either positive (pleasant) or neutral in the sleep-control (SC) and sleep-deprivation (SD) groups. A mixed-design ANOVA [between-subjectfactor of group (sleep control, sleep deprivation) and within-subjects factor of emotional response (pleasant, neutral)] demonstrated no significant main effect of group $\left(F_{(1,25)}=0.19\right.$, $p=0.67)$ and no significant main effect of emotional response $\left(F_{(1,25)}=2.02, p=0.16\right)$, but a significant group $\times$ emotional response interaction $\left(F_{(1,25)}=4.09, p=0.04\right.$; across group and condition comparison). Posthoctests within each group (symbol above bar) represent comparisons of each category to the null hypothesis of equal categorical assignment (50\%), with significance values provided in the main text. $\boldsymbol{B}$, The corresponding change in response tendency, characterized as positive bias score, represented as the subtracted difference in the proportion of positive items relative to the proportion of neutral items in the sleep-control and sleep-deprivation group. A zero value represents equal numbers of stimuli assigned to neutral and pleasant categories, while a positive score represents a greater proportion of stimuli rated as pleasant relative to neutral (and vice versa). Comparison reflects significance at $p<0.05\left({ }^{*}\right)$ and $\left.p<0.011^{* *}\right)$. n.s., Nonsignificant. Error bars represent s.e.m. Corresponding response times and analyses are provided in supplemental Table 1 (available at www.jneurosci.org as supplemental material). 
(both paired $t$ test, $\left.t_{(13)}>3.06, p<0.009\right)$. In contrast, no difference in the proportion of pleasant and neutral ratings was observed in the sleep-control group (both paired $t$ test, $t_{(12)}<0.63$, $p>0.54)$. This change in response tendency was further characterized within each subject by calculating a positive bias score, representing the subtracted difference in the proportion of positive items relative to the proportion of neutral items (Fig. $1 B$ ). Participants in the sleep-deprived group demonstrated a strong positive ratings bias, while those in the control group showed no such significant shift in response tendency, with these scores being significantly different between groups (Fig. $1 B$ ) (two-sample $t$ test, $t_{(25)}=2.17, p=0.04$ ). Note that the near-zero value in positive response bias score in the sleep-rested control group indicates that participants rated near-equal proportions of the stimuli as pleasant and neutral (and not a lack of discrimination between the images). Together, these data describe a lopsided bias in the proportion of positive emotional stimulus judgments in the sleep-deprived group relative to the control group.

\section{fMRI-activation differences}

We first contrasted brain activation associated with reactivity to the increasing positive stimulus gradient between the two groups. Supporting the original hypothesis, significantly greater activation was identified in the deprivation group in a restricted portion of a priori mesolimbic regions previously implicated in mediating reward-driven behavior, including an area consistent with the VTA of the brainstem (Adcock et al., 2006; Shohamy and Wagner, 2008; Düzel et al., 2009), and the left putamen of the dorsal striatum (Berridge and Robinson, 2003; Schultz, 2006; Hampton et al., 2007; Knutson and Wimmer, 2007; Valentin and O'Doherty, 2009) (Fig. 2A; coordinates provided in Table 1). In addition, increases in reactivity were also identified in a priori affect-associated regions of the amygdala and left insula cortex (Berridge and Robinson, 2003; Murray, 2007). Greater activation in the deprivation group was also identified in the visual processing pathway in the left fusiform gyrus, although this non-a priori region did not survive a more conservative statistical threshold correction (Table 1). Conversely, significantly less activation was observed in the sleep-deprivation group, relative to the control group, in mnemonic-related networks of the right posterior hippocampus and precuneus (Yoo et al., 2007a; Van Der Werf et al., 2009), as well as the left middle occipital gyrus (Fig. 2B), although these non-a priori regions also did not survive the more conservative statistical threshold correction (Table 1). That these increases in activation in the sleep-deprivation group were not due to a generalized or nonspecific amplified reactivity to any and all stimulus kinds was confirmed by a subset analysis of the upper quartile stimulus set (most positive) and lower quartile stimulus set (most neutral). The analyses, described in detail in Figure 3, demonstrate consistent between-group reactivity differences for the most positive stimulus set, but not the most neutral stimuli.

\section{fMRI-association with task performance}

We next sought to test our hypothesis that regions exhibiting significantly greater reactivity in sleep-deprived participants at the overall group level (described in Fig. 2) were common and hence overlapping with those regions that demonstrated a significant correlation with the positive bias score in the sleep-deprived group (described in Fig. $1 B$ ). We first identified regions of taskrelated brain activity that correlated with the positive emotional bias scores in the sleep-deprived group using a regression model, and subsequently contrasted them with the between-group re-

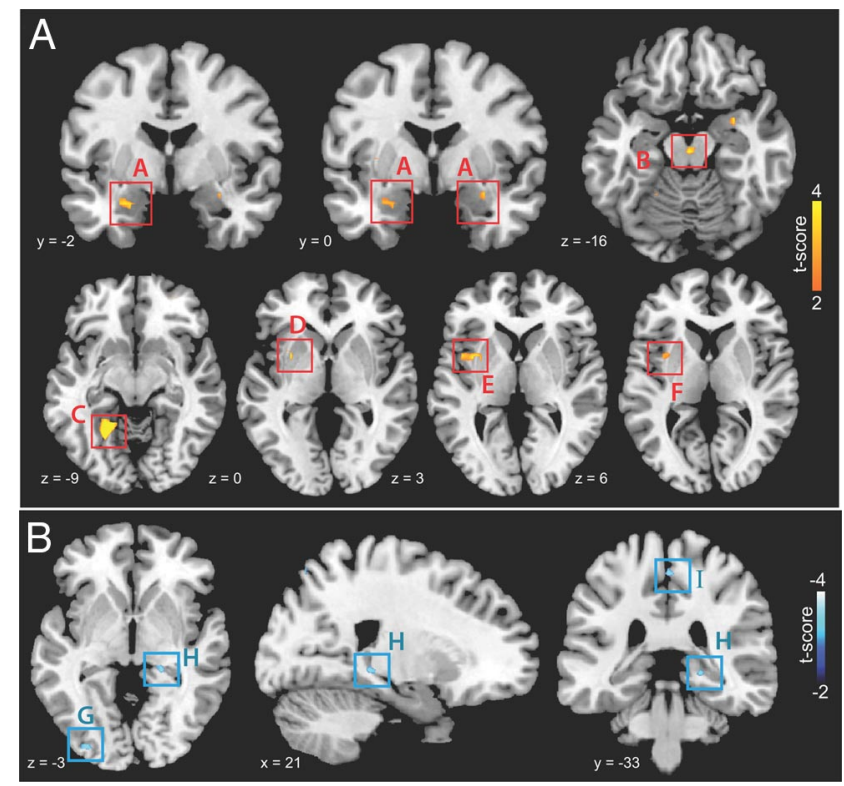

Figure 2. $\mathrm{fMRI}$ group-level activation differences. $\boldsymbol{A}, \boldsymbol{B}$, Statistical activation maps demonstrating regions of significantly increased activation (red box surround) in response to the positive emotion stimulus gradient in the sleep-deprivation group, relative to the sleep-control group, in the amygdala (A), VTA (B), fusiform gyrus (C), putamen (D and E), and insular cortex (D and $F)(A)$, and corresponding regions of significantly less activation (blue box surround) in the sleep-deprivation group, relative to the sleep-control group, in the occipital cortex (G), hippocampus $(\mathrm{H})$, and precuneus $(\mathrm{I})(\boldsymbol{B})$. Significant difference displayed on $\mathrm{T} 1$ anatomical slices (MNI slice number lower left). Histograms of parameter estimates (effect size) for averaged activity for regions of significant difference are provided in supplemental Figure 1 (available at www.jneurosci.org as supplemental material), with coordinates provided in Table 1. Images are displayed in neurological convention, with left side corresponding to left hemisphere. Effects are displayed at whole brain uncorrected cortex $p<0.001 ; \geq 5$ contiguous voxels, with corrected significance reported in Table 1.

gions of difference described in Figure 2. Overlaying the two contrasts - the group-level differences in activation between the two groups, and the regions correlating with the positive bias score in the sleep-deprivation group-demonstrated homology in several areas: the VTA, the left fusiform gyrus, and left insular cortex (Fig. 4A; coordinates provided in supplemental Table 2, available at www.jneurosci.org as supplemental material). A formal test of this homology was examined using conjunction analysis, confirming clusters of shared activity in all three of these regions (Fig. $4 B$; with equivalent analyses for the control group also reported in supplemental material and corresponding supplemental Fig. 3, available at www.jneurosci.org as supplemental material). Correlations between the positive bias score and brain activation within the sleep-control group are provided in supplemental Figure 3 (available at www.jneurosci.org as supplemental material) (coordinates in supplemental Table 2, available at www.jneurosci.org as supplemental material). Therefore, several a priori brain regions that significantly correlated with the positive bias score within the sleep-deprivation group were anatomically convergent with the same regions that were significantly different between the sleep-deprivation and sleep-control groups at the independent group level of analysis.

\section{fMRI-functional connectivity differences}

Focusing a priori on mesolimbic regions demonstrating significant difference at the group level (VTA, amygdala, insula, putamen), we finally sought to test our hypothesis that a differential network of functional connectivity was associated with activity 
in these target ROIs. For the VTA ROI, relative to the sleep-control group, significantly stronger functional connectivity was identified in the deprivation group in regions of the left amygdala and left anterior temporal pole (Fig. 5A). The insular cortex ROI demonstrated greater coupling with a number of visual cortex regions (Fig. 5B), together with an area at the left posterior insular cortex junction. The bilateral amygdala ROIs, which demonstrated increased reactivity in the deprivation condition at the overall group level, showed significant decreases in connectivity with medial prefrontal cortex and bilateral orbitofrontal cortex in the deprivation group, together with decreased coupling in the left fusiform gyrus (Fig. 5C). No between-group differences in connectivity were identified for the putamen ROI. While not a component of our original hypothesis, the fusiform area, which demonstrated greater reactivity in the sleep-deprived participants at the overall group level, exhibited stronger connectivity with the left anterior temporal pole, similar to the VTA, with additional regions of increased connectivity in left superior insula and left lateral prefrontal cortex (supplemental Fig. 4A, available at www.jneurosci.org as supplemental material). Connectivity results for regions that demonstrated less reactivity in the deprivation condition at the overall group level are provided in supplemental Figure $4 B$ (available at www. jneurosci.org as supplemental material). Therefore, in addition to enhanced reactivity in the VTA, insula, and amygdala in the deprivation group, differences in functional connectivity of these regions were also observed, associated with decreased coupling in midline prefrontal and orbitofrontal cortices, yet increased connectivity with related mesolimbic and early sensory perceptual regions.

\section{Discussion}

Here we demonstrate that sleep deprivation amplifies reactivity throughout select midbrain, striatal, limbic, and visual perceptual processing regions in response to positive emotional stimuli. Moreover, these regional increases were additionally associated with a reduction in functional connectivity in medial and orbitofrontal cortex, yet an enhanced coupling in early primary visual processing pathways and extended limbic regions. Behaviorally, these neural changes were accompanied by a significant bias in the number of stimuli rated as emotionally pleasant in the sleep-deprived group, the extent of
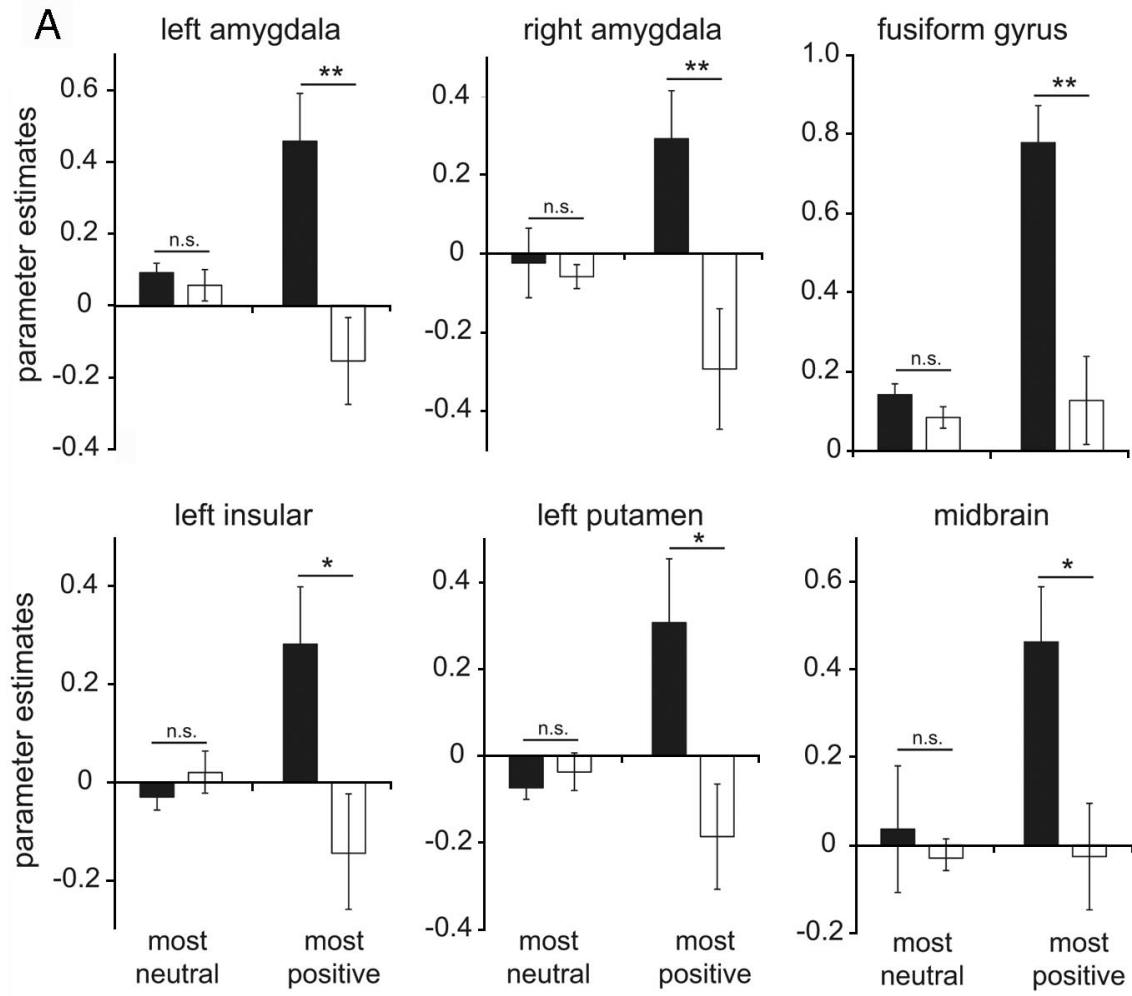

sleep deprivation group

sleep control group

B
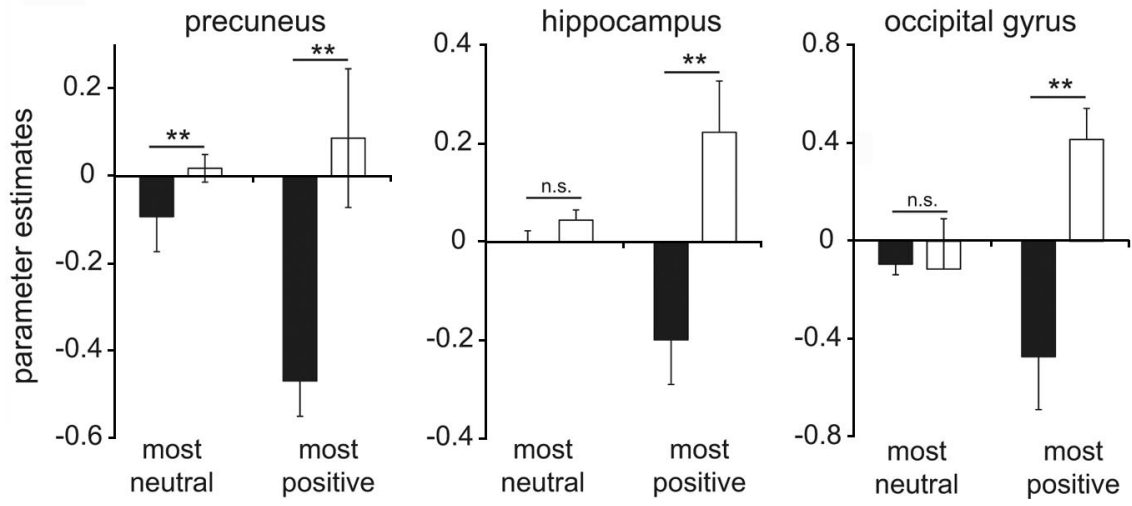

Figure 3. Histograms of parameter estimates (effect size) for nonparametrically modulated activity in regions of group-level difference, displayed in Figure 2, but for a subset analysis of the 25 most neutral and 25 most positive images. To determine whether increases in activation observed in the sleep-deprivation group were due to a generalized (nonspecific) amplified reactivity to all stimulus kinds, independent of emotion strength, a further subset analysis of the upper quartile stimulus set (most positive) and lower quartile stimulus set (most neutral) was performed for regions identified as different between the two groups. $A$, For all regions described as more reactive in the deprivation group (Fig. $2 A$ ), the subset analyses revealed similar activation levels between the two groups in response to the most neutral pictures, yet highly significant differences between groups for the most positive pictures. Between-group statistical $t$ test comparisons are provided within the figure. All within-group comparisons between the most neutral and most positive stimuli were significant in the deprivation group (all $p<0.04$ ), yet nonsignificant in the control group (all $p>0.12$ ), with the exception of activity in the right amygdala, which showed a trend toward significance $(p=0.07)$. Therefore, across all regions showing greater reactivity in the deprivation group across the entire stimulus set, a condition-specific relationship was observed, expressly in response to positive stimuli, while reactivity to more neutral images was similar in the two groups. $\boldsymbol{B}$, This was also true for the reduction in activity identified in the hippocampus and left occipital gyrus in the deprivation group. However, it was not the case for the remaining region showing decreased reactivity at the overall group level in the sleep-deprived participants - the precuneus - which demonstrated nonspecific reductions in reactivity for both neutral and negative stimulus subsets, relative to the control group. Between-group statistical $t$ test comparisons are provided within figure. All within-group comparisons between the most neutral and most positive stimuli were significant in the control group and deprivation group (all $p<0.03$ ), with the exception of activity in the precuneus in the control group ( $p=0.56$ ). All between group comparisons reflect significance at $p<0.05\left(^{*}\right)$ and $\left.p<0.005{ }^{* *}\right)$. Error bars represent s.e. 


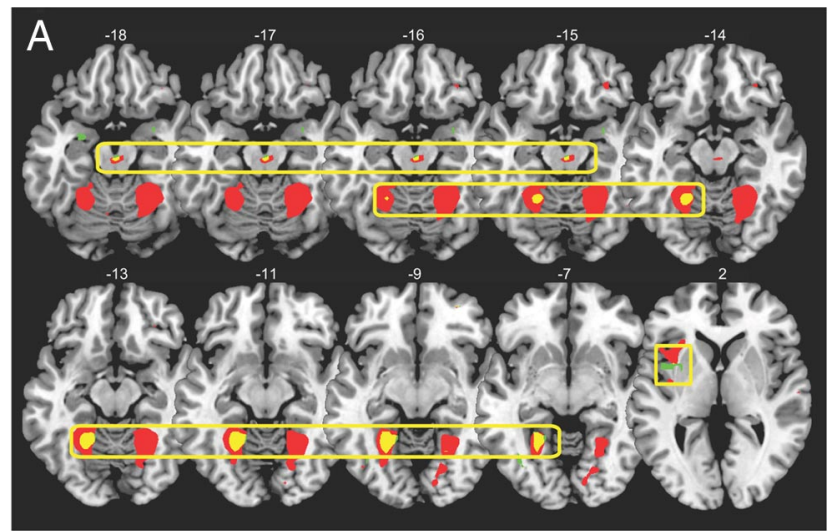

Group difference (Sleep Deprived > Sleep Control)

Correlation with positive bias score in the Deprivation Group Overlap

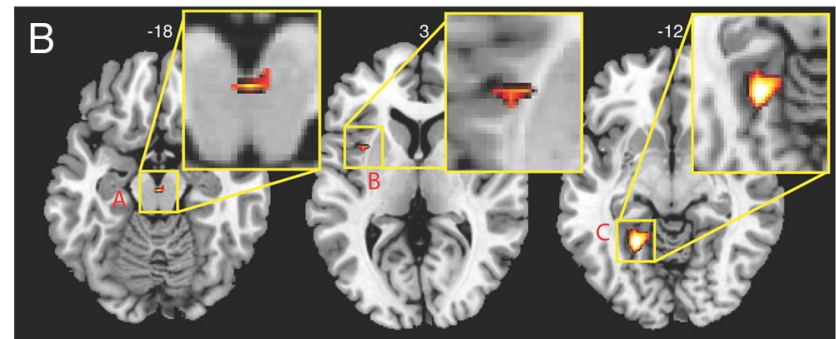

Figure 4. fMRI correlation and conjunction analyses. $\boldsymbol{A}$, Regions of task-related activation that were significantly more active in the sleep-deprivation group than in the sleep-control group at the overall group level (green; as described in Fig. $2 \mathrm{~A}$ ), together with regions showing a correlation with the positive bias score within the sleep-deprivation group (red, as above), and the overlap homology between the two (yellow with yellow box surround), identifying the insular, VTA, and fusiform gyrus. Coordinates are provided in supplemental Table 2 (available at www.jneurosci.org as supplemental material). $\boldsymbol{B}$, Results of the conjunction test confirming the overlap homology shown in Figure $3 B$ (yellow with yellow box surround) in a priori regions of the insular [A; peak MNI space coordinates $(x, y, z)$ : -37,7,2], the VTA (B; peak: $1,-21,-18$ ), (C; peak: $-24,-53,-12)$. Images are displayed in neurological convention on T1 anatomical axial slices (MNI slice number above), with left side corresponding to left hemisphere.

which correlated with overlapping regions in these reward- and affect-associated networks.

The striatum (including the caudate, nucleus accumbens, and putamen) has consistently been implicated in motivation and emotion regulation (Knutson and Cooper, 2005; Schultz, 2006), and can respond in a scaled, parametric way to increasing positive-reward valence (Knutson et al., 2000, 2001; Delgado et al., 2003). Striatal regions receive dopaminergic projections from the midbrain VTA, the modulatory effects of which are believed to be important in regulating the brain's response to positive rewards by aiding information integration within the striatum (Knutson and Cooper, 2005; Schultz, 2006). We found that a subset of regions in this dopaminergic reward network, encompassing the putamen and VTA, exhibited significantly greater activation in response to positive emotional stimuli in the sleepdeprived group, relative to those who had slept. This increase in reactivity of the VTA - a structure that can also scale in response magnitude with increasing reward value (Fields et al., 2007) may offer a putative mechanism underlying the increase in the biased number of positive stimulus judgments observed in the deprivation group. Supporting such a hypothesis, activation in the VTA was not only different between the two groups, but within the deprivation group was additionally correlated with the positive response bias scores across sleep-deprived partic- ipants (discussed further below). The putamen, situated in the dorsal striatum, has previously been linked to action-oriented components of reward-driven behavior that are stimulus relevant (rather than automated/habitual) (Balleine et al., 2007). Therefore, beyond necessarily coding reward value, which more ventral aspects of the striatum may represent (Knutson and Wimmer, 2007), the increased putamen activation identified in the deprivation group may reflect a stronger mapping to, or learned association with, the positive stimulus response choices.

The enhanced mesolimbic reactivity identified in the deprivation group complements recent investigations by Volkow and colleagues using PET [11C]raclopride binding-a dopamine $\mathrm{D}_{2} / \mathrm{D}_{3}$ receptor radioligand-describing increases in dopamine activity within reward networks following acute sleep loss, including the striatum (Volkow et al., 2008, 2009). Our findings also converge with studies in rodents describing a heightened sensitivity of dopaminergic receptors following sleep restriction (Tufik et al., 1978; Tufik, 1981; Troncone et al., 1988). Together with our own findings, this collection of reports offers an emerging view that acute sleep deprivation may induce enhanced activity throughout the mesolimbic reward system at both anatomical and dopaminergic neurochemical levels.

In addition to the increase in the VTA and dorsal striatum, the amygdala and the insular cortex also exhibited amplified reactivity in the deprivation group in response to the positive emotional stimulus gradient. Both the amygdala and the insular cortex can preferentially respond to emotional salience, playing a role not only in registering negative valence, but also in reactivity toward, and coding of, positive stimuli (Berridge and Robinson, 2003; Murray, 2007). Indeed, this collection of limbic regions, together with the VTA and striatum, has been proposed as a broad network supporting motivation and reward processing (Camara et al., 2009). Our findings further support the hypothesis that sleep loss is not exclusively associated with enhanced emotional brain reactivity toward threat-relevant negative stimuli (Yoo et al., 2007b; Franzen et al., 2009), but also appears to impose a labile, bidirectional nature of affective imbalance, including increased neural and behavioral reactivity to positive experiences. The sleep-deprived state may therefore represent a pendulum-like emotional circumstance associated with amplified reactivity across the full range of affective valence (both positive and negative), as has been proposed in insomnia (Epstein and Bootzin, 2002), and potentially contributing to reported deficits in judgment and decision making following sleep loss (Alexopoulos et al., 1988; Killgore et al., 2006, 2007; McKenna et al., 2007; Venkatraman et al., 2007; Holm et al., 2009). Based on previous findings demonstrating increased amygdala reactivity to negatively valenced stimuli following sleep deprivation (Yoo et al., 2007b), the increased amygdala reactivity identified in the current study, unlike the VTA, putamen, and insular cortex, may not be selective to positive valence per se (summarized in supplemental Fig. 5, available at www.jneurosci.org as supplemental material). A shared property of both the positive valence stimuli in the current study and the negative valence stimuli in previous work (Yoo et al., 2007b) is high arousal. Future investigations that manipulate both valence and arousal in the same experiment will help dissociate between these possibilities.

In addition to group-level differences in brain activation, participants in the deprivation group, unlike the control group, demonstrated an overlapping correlation between the positive emotion bias score and regions associated with incentive processing: the VTA and the insula. Therefore, reward-sensitive areas 

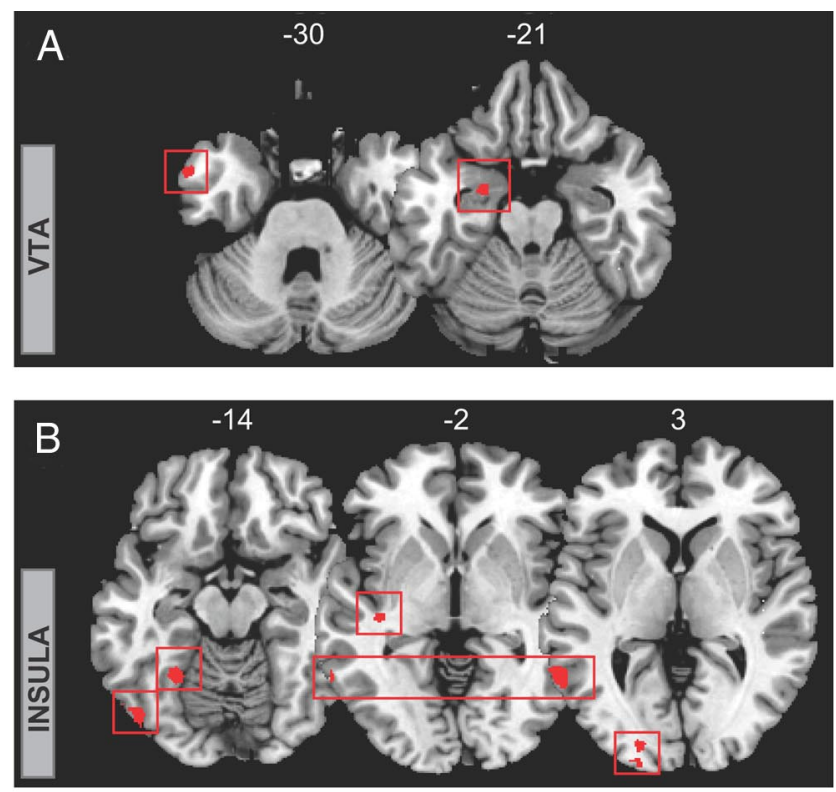

Sleep Deprivation > Sleep Control $\square$ Sleep Control > Sleep Deprived

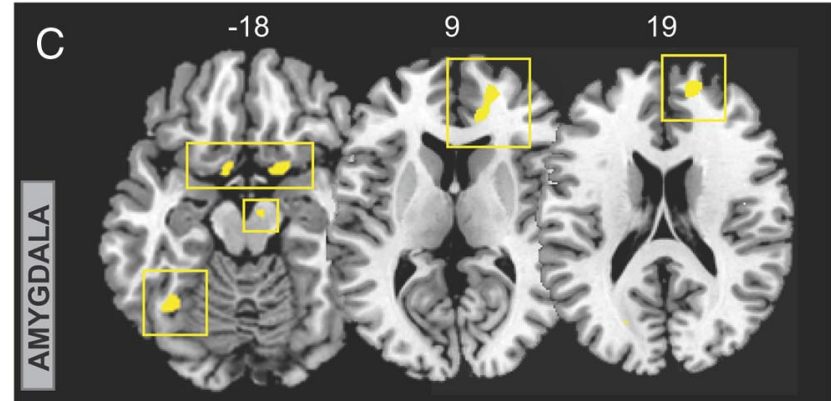

Figure 5. Group-level differences in functional connectivity. $A, B$, Regions showing significantly greater functional connectivity (red with red box surround) in the sleep-deprivation group relative to the sleep-control group for ROIs in the VTA (seed), in the left anterior temporal pole (MNI peak: -57 , $6,-30 ; Z$ score $=3.74)$ and left amygdala (peak: $-24,-6,-24 ; Z$ score $=3.93)(A)$; and the insular (seed), in the left lateral occipital cortex (peak: $-48,-75,-9 ; Z$ score $=3.63$ ), medial fusiform gyrus (peak: $-27,-54,-15 ; Z$ score $=3.98$ ), posterior insular cortex (peak: $-39,-24$, $-3 ; Z$ score $=4.02)$, posterior middle temporal lobe (peak: $-63,-54,3 ; Z$ score $=3.88$ ), and left occipital cortex (peak: $-21,-102,3 ; Z$ score $=3.82)(B)$. C, Regions showing significantly less functional connectivity (yellow with yellow box surround) in the sleep-deprivation group relative to the sleep-control group for bilateral amygdala ROIs in (leftseed) the left orbitofrontal cortex (peak: 18, $9,-18 ; Z$ score $=4.12$ ), right orbitofrontal cortex (peak: $-9,6,-15 ; Z$ score $=3.51$ ), medial prefrontal cortex (peaks: 15, 54, 18, Zscore $=4.02 ; 21,51,6, Z$ score $=3.71 ; 15,39,9, Z$ score $=$ 3.42), left fusiform gyrus (peak: $-36,-57,-18 ; Z$ score $=3.72$ ), and (right seed) the midbrain (peak: $9,-12,-18 ; Z$ score $=3.35$ ). Images are displayed in neurological convention on $\mathrm{T} 1$ anatomical axial slices (MNI slice number above), with left side corresponding to left hemisphere.

that were more active in the sleep-deprivation group were additionally correlated with individual differences in positive response bias score across the deprived participants. In line with models suggesting that affect-sensitive regions may interact bidirectionally with object processing in early sensory cortex (Rolls and Grabenhorst, 2008), we also identified significantly greater reactivity in the fusiform gyrus in sleep-deprived relative to sleeprested subjects, which similarly showed a conjunctive overlap with the positive response bias score in sleep-deprived participants. Such enhancement of activation in unimodal cortices additionally parallels findings describing sensitization of early visual processing areas to incoming stimuli during expectation of salient or rewarding experiences (Padmala and Pessoa, 2008). The lack of correlation between the positive bias score and reward-related areas in the sleep-control group may be related to the limited degree of positive bias expressed by these participants. Alternatively, under sleep-rested conditions, with appropriate prefrontal regulatory control maintained, these reward networks may not be significantly engaged, even in those with more pronounced positive bias response tendencies.

Functional connectivity analyses based on seed regions in the VTA, insula, and amygdala identified more strongly interconnected relationships among affect-associated networks in the sleep-deprived group. Specifically, the VTA demonstrated stronger connectivity to the amygdala and the anterior temporal pole [the latter also a region previously implicated in positive emotion processing (Pelletier et al., 2003)], while the insula exhibited stronger coupling with early visual perceptual regions and the posterior insula cortex. This may indicate the existence of a "closed loop" of reactivity in response to positive emotional experiences under conditions of sleep deprivation, reciprocally facilitating activation throughout a network of regions known to respond to pleasurable stimuli. Moreover, and in combination with the group-level differences identified in the fusiform gyrus, the increases in connectivity between these reward-associated networks and early sensory perceptual regions may enhance attention to features of the emotional objects, further increasing their perceived salience (Rolls and Grabenhorst, 2008).

Despite the increased reactivity throughout reward-relevant brain networks in the sleep-deprived group, these enhancements were not associated with greater connectivity in $\mathrm{mPFC}$ or orbitofrontal (OFC) networks, which often couple with these subcortical systems (e.g., Schultz, 2006; Hampton et al., 2007; Knutson and Wimmer, 2007; Wallis, 2007; Rolls and Grabenhorst, 2008). Indeed, the enhanced reactivity in the amygdala in the sleepdeprived group was associated with significant reductions in connectivity with bilateral OFC and right $\mathrm{mPFC}$ regions. Decreased amygdala-mPFC connectivity has also been demonstrated under conditions of sleep deprivation in response to negative stimuli (Yoo et al., 2007b), again leading to the speculation that such impairment is potentially arousal- rather than valence-specific (supplemental Fig. 5, available at www.jneurosci.org as supplemental material). However, this seems not to be the case for changes in OFC connectivity, which were selectively identified in the current study using positive valence stimuli, and not evident in previous work using negative valence stimuli (Yoo et al., 2007b). More generally, this paucity of connectivity is of potential relevance, considering that OFC as well as mPFC regions can aid in coding and representing parameters of value and risk during positive, rewarding experiences (Wallis, 2007; Rolls and Grabenhorst, 2008). A lack of appropriate engagement of these limbic-related PFC regions may therefore contribute to altered positive emotional sensitivity and regulatory control under conditions of sleep loss, with cognition being driven more by subcortical affective circuits (Killgore et al., 2006, 2007; Venkatraman et al., 2007). Such a notion would support the concept of PFC sensitivity to, and hence disinhibition induced by, a lack of sleep (Harrison et al., 2000; Yoo et al., 2007b).

Finally, and while remaining speculative, our current findings may afford clinical insights into the neural mechanisms supporting the fast-acting mood improvement triggered by acute sleep deprivation in depressed patients (Wirz-Justice and Van den Hoofdakker, 1999), namely, enhanced reactivity to pleasurable experiences, mediated by amplified neural reactivity and altered connectivity throughout affective and reward brain networks. This hypothesis would fit well with the core symptom of anhedonia in depression, preventing the ability to experience positive 
emotion in response to normally pleasurable events. In addition to resting brain activity (Wu et al., 1999; Clark et al., 2006), subsequent clinical studies investigating event-related brain reactivity in response to positive emotional stimuli in depressed patients undergoing sleep deprivation may offer useful insights in determining the neural basis of this treatment's benefit.

\section{References}

Adcock RA, Thangavel A, Whitfield-Gabrieli S, Knutson B, Gabrieli JD (2006) Reward-motivated learning: mesolimbic activation precedes memory formation. Neuron 50:507-517.

Alexopoulos GS, Abrams RC, Young RC, Shamoian CA (1988) Cornell scale for depression in dementia. Biol Psychiatry 23:271-284.

Armitage R (2007) Sleep and circadian rhythms in mood disorders. Acta Psychiatr Scand Suppl 104-115.

Balleine BW, Delgado MR, Hikosaka O (2007) The role of the dorsal striatum in reward and decision-making. J Neurosci 27:8161-8165.

Benca RM, Obermeyer WH, Thisted RA, Gillin JC (1992) Sleep and psychiatric disorders. A meta-analysis. Arch Gen Psychiatry 49:651-668; discussion 669-670.

Berridge KC, Robinson TE (2003) Parsing reward. Trends Neurosci 26:507-513.

Bliss EL, Clark LD, West CD (1959) Studies of sleep deprivationrelationship to schizophrenia. AMA Arch Neurol Psychiatry 81:348-359.

Büchel C, Holmes AP, Rees G, Friston KJ (1998) Characterizing stimulusresponse functions using nonlinear regressors in parametric fMRI experiments. Neuroimage 8:140-148.

Camara E, Rodriguez-Fornells A, Ye Z, Münte TF (2009) Reward networks in the brain as captured by connectivity measures. Front Neurosci 3:350-362.

Chib VS, Rangel A, Shimojo S, O'Doherty JP (2009) Evidence for a common representation of decision values for dissimilar goods in human ventromedial prefrontal cortex. J Neurosci 29:12315-12320.

Clark CP, Brown GG, Archibald SL, Fennema-Notestine C, Braun DR, Thomas LS, Sutherland AN, Gillin JC (2006) Does amygdalar perfusion correlate with antidepressant response to partial sleep deprivation in major depression? Psychiatry Res 146:43-51.

Dahl RE (1996) Regulation of sleep and arousal: development and psychopathology. Dev Psychopathol 8:3-27.

D'Ardenne K, McClure SM, Nystrom LE, Cohen JD (2008) BOLD responses reflecting dopaminergic signals in the human ventral tegmental area. Science 319:1264-1267.

Delgado MR, Locke HM, Stenger VA, Fiez JA (2003) Dorsal striatum responses to reward and punishment: effects of valence and magnitude manipulations. Cogn Affect Behav Neurosci 3:27-38.

Delgado MR, Miller MM, Inati S, Phelps EA (2005) An fMRI study of reward-related probability learning. Neuroimage 24:862-873.

Dinges DF, Pack F, Williams K, Gillen KA, Powell JW, Ott GE, Aptowicz C, Pack AI (1997) Cumulative sleepiness, mood disturbance, and psychomotor vigilance performance decrements during a week of sleep restricted to 4-5 hours per night. Sleep 20:267-277.

Düzel E, Bunzeck N, Guitart-Masip M, Wittmann B, Schott BH, Tobler PN (2009) Functional imaging of the human dopaminergic midbrain. Trends Neurosci 32:321-328.

Epstein DR, Bootzin RR (2002) Insomnia. Nurs Clin North Am 37:611-631.

Fields HL, Hjelmstad GO, Margolis EB, Nicola SM (2007) Ventral tegmental area neurons in learned appetitive behavior and positive reinforcement. Annu Rev Neurosci 30:289-316.

Franzen PL, Buysse DJ, Dahl RE, Thompson W, Siegle GJ (2009) Sleep deprivation alters pupillary reactivity to emotional stimuli in healthy young adults. Biol Psychol 80:300-305.

Friston KJ, Holmes AP, Worsley KJ, Poline JB, Frith CD, Frackowiak RS (1995) Statistical parametric maps in functional imaging: a general linear approach. Hum Brain Mapp 2:189-210.

Friston KJ, Buechel C, Fink GR, Morris J, Rolls E, Dolan RJ (1997) Psychophysiological and modulatory interactions in neuroimaging. Neuroimage $6: 218-229$.

Friston KJ, Glaser DE, Henson RN, Kiebel S, Phillips C, Ashburner J (2002) Classical and Bayesian inference in neuroimaging: applications. Neuroimage 16:484-512.

Gillin JC, Buchsbaum M, Wu J, Clark C, Bunney W Jr (2001) Sleep deprivation as a model experimental antidepressant treatment: findings from functional brain imaging. Depress Anxiety 14:37-49.
Gottfried JA, O'Doherty J, Dolan RJ (2003) Encoding predictive reward value in human amygdala and orbitofrontal cortex. Science 301:1104-1107.

Guitart-Masip M, Bunzeck N, Stephan KE, Dolan RJ, Düzel E (2010) Contextual novelty changes reward representations in the striatum. J Neurosci 30:1721-1726.

Hampton AN, Adolphs R, Tyszka MJ, O’Doherty JP (2007) Contributions of the amygdala to reward expectancy and choice signals in human prefrontal cortex. Neuron 55:545-555.

Harrison Y, Horne JA, Rothwell A (2000) Prefrontal neuropsychological effects of sleep deprivation in young adults—a model for healthy aging? Sleep 23:1067-1073.

Harvey AG (2008) Sleep and circadian rhythms in bipolar disorder: seeking synchrony, harmony, and regulation. Am J Psychiatry 165:820-829.

Holm SM, Forbes EE, Ryan ND, Phillips ML, Tarr JA, Dahl RE (2009) Reward-related brain function and sleep in pre/early pubertal and mid/ late pubertal adolescents. J Adolesc Health 45:326-334.

Horne JA (1993) Human sleep, sleep loss and behaviour. Implications for the prefrontal cortex and psychiatric disorder. Br J Psychiatry 162:413-419.

Karama S, Lecours AR, Leroux JM, Bourgouin P, Beaudoin G, Joubert S, Beauregard M (2002) Areas of brain activation in males and females during viewing of erotic film excerpts. Hum Brain Mapp 16:1-13.

Killgore WD, Balkin TJ, Wesensten NJ (2006) Impaired decision making following $49 \mathrm{~h}$ of sleep deprivation. J Sleep Res 15:7-13.

Killgore WD, Killgore DB, Day LM, Li C, Kamimori GH, Balkin TJ (2007) The effects of 53 hours of sleep deprivation on moral judgment. Sleep 30:345-352.

Knutson B, Cooper JC (2005) Functional magnetic resonance imaging of reward prediction. Curr Opin Neurol 18:411-417.

Knutson B, Wimmer GE (2007) Splitting the difference: how does the brain code reward episodes? Ann N Y Acad Sci 1104:54-69.

Knutson B, Westdorp A, Kaiser E, Hommer D (2000) FMRI visualization of brain activity during a monetary incentive delay task. Neuroimage 12:20-27.

Knutson B, Adams CM, Fong GW, Hommer D (2001) Anticipation of increasing monetary reward selectively recruits nucleus accumbens. J Neurosci 21:RC159.

Knutson B, Taylor J, Kaufman M, Peterson R, Glover G (2005) Distributed neural representation of expected value. J Neurosci 25:4806-4812.

Kuhl BA, Shah AT, DuBrow S, Wagner AD (2010) Resistance to forgetting associated with hippocampus-mediated reactivation during new learning. Nat Neurosci 13:501-506.

Lang PJ (1997) International Affective Picture System (IAPS): technical manual and affective ratings. Gainesville, FL: University of Florida.

Levin R, Nielsen TA (2009) Nightmares, bad dreams, and emotion dysregulation. A review and new neurocognitive model of dreaming. Curr Dir Psychol Sci 18:84-88.

Lieberman MD, Cunningham WA (2009) Type I and type II error concerns in fMRI research: re-balancing the scale. Soc Cogn Affect Neurosci 4:423-428.

McKenna BS, Dickinson DL, Orff HJ, Drummond SP (2007) The effects of one night of sleep deprivation on known-risk and ambiguous-risk decisions. J Sleep Res 16:245-252.

Murray EA (2007) The amygdala, reward and emotion. Trends Cogn Sci 11:489-497.

Nichols T, Brett M, Andersson J, Wager T, Poline JB (2005) Valid conjunction inference with the minimum statistic. Neuroimage 25:653-660.

Padmala S, Pessoa L (2008) Affective learning enhances visual detection and responses in primary visual cortex. J Neurosci 28:6202-6210.

Pelletier M, Bouthillier A, Lévesque J, Carrier S, Breault C, Paquette V, Mensour B, Leroux JM, Beaudoin G, Bourgouin P, Beauregard M (2003) Separate neural circuits for primary emotions? Brain activity during selfinduced sadness and happiness in professional actors. Neuroreport 14:1111-1116.

Pochon JB, Levy R, Fossati P, Lehericy S, Poline JB, Pillon B, Le Bihan D, Dubois B (2002) The neural system that bridges reward and cognition in humans: an fMRI study. Proc Natl Acad Sci U S A 99:5669-5674.

Rolls ET, Grabenhorst F (2008) The orbitofrontal cortex and beyond: from affect to decision-making. Prog Neurobiol 86:216-244.

Sabatinelli D, Bradley MM, Lang PJ, Costa VD, Versace F (2007) Pleasure rather than salience activates human nucleus accumbens and medial prefrontal cortex. J Neurophysiol 98:1374-1379.

Schultz W (2006) Behavioral theories and the neurophysiology of reward. Annu Rev Psychol 57:87-115.

Shohamy D, Wagner AD (2008) Integrating memories in the human 
brain: hippocampal-midbrain encoding of overlapping events. Neuron 60:378-389.

Smith AP, Stephan KE, Rugg MD, Dolan RJ (2006) Task and content modulate amygdala-hippocampal connectivity in emotional retrieval. Neuron 49:631-638.

Sterpenich V, Albouy G, Boly M, Vandewalle G, Darsaud A, Balteau E, Dang-Vu TT, Desseilles M, D’Argembeau A, Gais S, Rauchs G, Schabus M, Degueldre C, Luxen A, Collette F, Maquet P (2007) Sleep-related hippocampo-cortical interplay during emotional memory recollection. PLoS Biol 5:e282.

Troncone LR, Ferreira TM, Braz S, Silveira Filho NG, Tufik S (1988) Reversal of the increase in apomorphine-induced stereotypy and aggression in REM sleep deprived rats by dopamine agonist pretreatments. Psychopharmacology (Berl) 94:79-83.

Tufik S (1981) Changes of response to dopaminergic drugs in rats submitted to REM-sleep deprivation. Psychopharmacology (Berl) 72:257-260.

Tufik S, Lindsey CJ, Carlini EA (1978) Does REM sleep deprivation induce a supersensitivity of dopaminergic receptors in the rat brain? Pharmacology 16:98-105.

Valentin VV, O'Doherty JP (2009) Overlapping prediction errors in dorsal striatum during instrumental learning with juice and money reward in the human brain. J Neurophysiol 102:3384-3391.

Van Der Werf YD, Altena E, Schoonheim MM, Sanz-Arigita EJ, Vis JC, De Rijke W, Van Someren EJ (2009) Sleep benefits subsequent hippocampal functioning. Nat Neurosci 12:122-123.

Venkatraman V, Chuah YM, Huettel SA, Chee MW (2007) Sleep deprivation elevates expectation of gains and attenuates response to losses following risky decisions. Sleep 30:603-609.

Volkow ND, Wang GJ, Telang F, Fowler JS, Logan J, Wong C, Ma J, Pradhan K, Tomasi D, Thanos PK, Ferré S, Jayne M (2008) Sleep deprivation decreases binding of $\left[{ }^{11} \mathrm{C}\right]$ raclopride to dopamine $\mathrm{D}_{2} / \mathrm{D}_{3}$ receptors in the human brain. J Neurosci 28:8454-8461.
Volkow ND, Tomasi D, Wang GJ, Telang F, Fowler JS, Wang RL, Logan J, Wong C, Jayne M, Swanson JM (2009) Hyperstimulation of striatal D2 receptors with sleep deprivation: implications for cognitive impairment. Neuroimage 45:1232-1240.

Walker MP, van der Helm E (2009) Overnight therapy? The role of sleep in emotional brain processing. Psychol Bull 135:731-748.

Wallis JD (2007) Orbitofrontal cortex and its contribution to decisionmaking. Annu Rev Neurosci 30:31-56.

Wirz-Justice A, Van den Hoofdakker RH (1999) Sleep deprivation in depression: what do we know, where do we go? Biol Psychiatry 46:445-453.

Wittmann BC, Schott BH, Guderian S, Frey JU, Heinze HJ, Düzel E (2005) Reward-related FMRI activation of dopaminergic midbrain is associated with enhanced hippocampus-dependent long-term memory formation. Neuron 45:459-467.

Wittmann BC, Daw ND, Seymour B, Dolan RJ (2008a) Striatal activity underlies novelty-based choice in humans. Neuron 58:967-973.

Wittmann BC, Schiltz K, Boehler CN, Düzel E (2008b) Mesolimbic interaction of emotional valence and reward improves memory formation. Neuropsychologia 46:1000-1008

Wu J, Buchsbaum MS, Gillin JC, Tang C, Cadwell S, Wiegand M, Najafi A, Klein E, Hazen K, Bunney WE Jr, Fallon JH, Keator D (1999) Prediction of antidepressant effects of sleep deprivation by metabolic rates in the ventral anterior cingulate and medial prefrontal cortex. Am J Psychiatry 156:1149-1158.

Yoo SS, Hu PT, Gujar N, Jolesz FA, Walker MP (2007a) A deficit in the ability to form new human memories without sleep. Nat Neurosci 10:385-392.

Yoo SS, Gujar N, Hu P, Jolesz FA, Walker MP (2007b) The human emotional brain without sleep - a prefrontal amygdala disconnect. Curr Biol 17:R877-R878.

Zohar D, Tzischinsky O, Epstein R, Lavie P (2005) The effects of sleep loss on medical residents' emotional reactions to work events: a cognitiveenergy model. Sleep 28:47-54. 